\title{
PERCEPÇÃO DA EQUIPE DE ENFERMAGEM ACERCA DA AVALIAÇÃO DA DOR EM RECÉM-NASCIDOS PREMATUROS
}

\section{PERCEPTION OF THE NURSING TEAM ABOUT PAIN EVALUATION IN PREMATURE NEWBORNS}

\section{PERCEPCIÓN DEL EQUIPO DE ENFERMERÍA EN LA EVALUACIÓN DEL DOLOR EN RECIÉN NACIDOS PREMATUROS}

\author{
Silas Santos Carvalho ${ }^{1}$, Janaína de Almeida Soares ${ }^{2}$, Jonathas Azevedo Pinheiro ${ }^{3}$, Melânia \\ dos Santos Queiroz ${ }^{4}$
}

Como citar esse artigo: Carvalho SS, Soares JA, Pinheiro JA, Queiroz MS. Percepção da equipe de enfermagem acerca da avaliação da dor em recém-nascidos prematuros. Rev Enferm Atenção Saúde [Internet]. 2021 [acesso em: ]; 10(2):e202117. doi: https://doi.org/ 10.18554/reas.v10i2.4281

\section{RESUMO}

Objetivo: Compreender as ações do enfermeiro na avaliação e no manejo da dor nos recémnascidos prematuros internados em uma unidade de terapia intensiva neonatal. Método: Estudo descritivo de abordagem qualitativa, desenvolvido com sete enfermeiras atuantes em uma maternidade. Os dados foram obtidos por meio de entrevista semiestruturada e as informações analisadas pelo método de análise de conteúdo temática. O projeto foi aprovado pelo Comitê de Ética em Pesquisa. Resultados: A partir da análise emergiram-se duas categorias temáticas: "Como o enfermeiro avalia a dor no recém-nascido prematuro" e "Ações do enfermeiro diante da dor no recém-nascido prematuro". As enfermeiras afirmaram terem percepção de situações dolorosas, porém, nota-se que sua identificação ocorria de forma fragmentada e superficial. As técnicas empregadas no estudo evidenciaram a necessidade de estabelecer uma sistemática na assistência. Conclusão: $\mathrm{Na}$ equipe de enfermagem ainda existe uma grande distância entre o conhecimento teórico, o uso de escalas e a conduta prática quanto à avaliação e manejo da dor de recém-nascidos prematuros.

Descritores: Dor; Recém-nascido prematuro; Enfermeiros; Unidade de terapia intensiva neonatal.

\footnotetext{
1 Enfermeiro. Mestre em Saúde Coletiva. Especialista em Enfermagem Obstétrica e Saúde da Mulher. Universidade Estadual de Feira de Santana-(UEFS), Feira de Santana-BA. Universidade Estadual de Feira de Santana, Feira de Santana-BA. http://orcid.org/0000-0001-8845-8902. ssc.academico@ hotmail.com

${ }^{2}$ Enfermeira. Pós graduanda em Enfermagem Obstétrica. Faculdade Anísio Teixeira (FAT)- Feira de SantanaBA. Feira de Santana-BA. almeida_jana@outlook.com

3 Enfermeiro. Faculdade Anísio Teixeira (FAT) Feira de Santana-BA. Feira de Santana-BA. jonathas_azevedo@live.com

${ }^{4}$ Enfermeira. Pós graduanda em Urgência, Emergência e UTI e Enfermagem Obstétrica. Faculdade Anísio Teixeira (FAT)- Feira de Santana-BA. Feira de Santana-BA. http://orcid.org/0000-0003-1931-3077. melania_queiroz@hotmail.com
} 


\begin{abstract}
Objective: To understand the actions of nurses in the assessment and management of pain in premature newborns admitted to a neonatal intensive care unit. Method: Descriptive study with a qualitative approach, developed with seven nurses working in a maternity hospital. The data were obtained through semi-structured interviews and the information was analyzed using the thematic content analysis method. The project was approved by the Research Ethics Committee. Results: From the analysis, two thematic categories emerged: "How the nurse assesses pain in the premature newborns" and "Actions of the nurse in the face of pain in the premature newborns". The nurses stated that they were aware of painful situations, however, it is noted that their identification occurred in a fragmented and superficial way. The techniques employed study showed the need to establish a system of assistance. Conclusion: In the nursing team, there is still a great distance between theoretical knowledge, the use of scales and practical conduct regarding the assessment and management of pain in premature newborns.
\end{abstract}

Descriptors: Pain; Infant premature; Nurses; Neonatal intensive care unit.

\title{
RESUMEN
}

Objetivo: Conocer las acciones del enfermero en la evaluación y manejo del dolor en recién nacidos prematuros ingresados en una unidad de cuidados intensivos neonatales. Método: Estudio descriptivo con abordaje cualitativo, desarrollado con siete enfermeras que laboran en una maternidad. Los datos se obtuvieron a través de entrevistas semiestructuradas y la información se analizó mediante el método de análisis de contenido temático. El proyecto fue aprobado por el Comité de Ética en Investigación. Resultados: Del análisis surgieron dos categorías temáticas: "Cómo valora el enfermero el dolor en el recién nacidos prematuos" y "Acciones del enfermero ante el dolor en el recién nacido prematuro". Las enfermeras manifestaron conocer situaciones dolorosas, sin embargo, se observa que su identificación ocurrió de manera fragmentada y superficial. Las técnicas empleadas estudio mostraron la necesidad de establecer un sistema de asistencia. Conclusión: En el equipo de enfermería, aún existe una gran distancia entre los conocimientos teóricos, el uso de escalas y la conducta práctica en la evaluación y manejo del dolor en recién nacidos prematuros.

Descriptores: Dolor; Recién nacido prematuro; Enfermeros; Unidades de cuidado intensive neonatal.

\section{INTRODUÇÃO}

A prematuridade é um fator que eleva as taxas de morte no período neonatal, originando agravos de difícil mensuração aos recém-nascidos $(\mathrm{RN})$. Aqueles que evoluem com um bom prognóstico, conseguem superar esse momento inicial da vida. $^{1} \mathrm{O}$ termo prematuridade é definido como o nascimento antes de 37 semanas de gestação e suas consequências são inúmeras, como: baixo peso ao nascer, problemas respiratórios, oculares, atrasos no desenvolvimento motor, imaturidade de órgãos e sistemas. ${ }^{1}$

Acreditou-se por muito tempo que os $\mathrm{RN}$, principalmente os pré-termos, não possuíam a capacidade de sentir dor, devido ao seu baixo desenvolvimento neurológico e sua inexpressividade de 
comunicação. $^{2}$ Identificou-se em um estudo que o RN hospitalizado pode passar por 50 a 150 procedimentos dolorosos diariamente. ${ }^{3}$

A dor pode ser estabelecida como uma sensação subjetiva e individual, relacionada a uma lesão tecidual real ou potencial. Quando se aborda o período neonatal, essa avaliação pode ser classificada em dois parâmetros: os comportamentais e os fisiológicos. O primeiro engloba as expressões faciais, a movimentação corporal e o choro; para o segundo, destacam-se as mudanças no ritmo cardíaco e respiratório, pressão arterial sistêmica e saturação de oxigênio. ${ }^{4}$

Para que a avaliação da dor seja efetiva são necessários instrumentos capazes de garantir a compreensão das manifestações desencadeadas pelo RN. São utilizadas escalas que se caracterizam pela obtenção de parâmetros, expressadas em mudanças fisiológicas e comportamentais, que se baseiam em determinadas expressões apresentadas após um estímulo doloroso. ${ }^{5,6}$

Para avaliar e promover o adequado manejo da dor por meio de tratamentos farmacológicos e não farmacológicos, a equipe de enfermagem deve ter conhecimento suficiente para que os cuidados prestados ao neonato sejam com qualidade. Assim, o presente estudo inserese na proposta da busca de medidas que avaliem e minimizem a dor do $\mathrm{RN}$ pelos enfermeiros em unidades de terapia intensiva neonatal (UTIN), visando modificações no padrão de assistência, na perspectiva da prática baseada em evidência, e sensibilizando aos profissionais de enfermagem quanto à avaliação e manejo da dor neonatal. Pretende-se assim, dar subsídios a futuros estudos e ações nos serviços de saúde em favor da melhoria da assistência neonatal em relação ao manejo adequado da dor.

Considerando a importância de uma avaliação adequada da dor bem como do manejo da mesma em recém-nascidos e dos possíveis sofrimentos que podem ser atenuados ou prevenidos, esse estudo levantou o seguinte problema: "Como é realizada a avaliação da dor e o manejo da mesma pelo enfermeiro em RN prematuros?" e teve como objetivo compreender as ações do enfermeiro na avaliação e no manejo da dor nos recémnascidos prematuros internados em uma UTIN.

\section{MÉTODOS}

Estudo do tipo exploratório, descritivo e com abordagem qualitativa, realizado em uma maternidade pública de um município do interior da Bahia, Brasil.

Foram selecionados para esse estudo os enfermeiros assistenciais da UTIN que aceitaram participar voluntariamente. Os 
critérios de exclusão foram enfermeiros que se apresentaram afastados por motivo de licença, férias ou não estiveram presentes durante o período da coleta de dados. A amostra do estudo foi constituída por 07 enfermeiras. Não foi realizado um levantamento prévio para identificar o número potencial de participantes. Este foi determinado de acordo com os princípios de saturação, ou seja, depoimentos sem novas informações.

A coleta de dados ocorreu em maio de 2017, por meio de entrevista semiestruturada que conteve questões sobre dados pessoais dos participantes e o conhecimento técnico e prático acerca do manejo da dor do $\mathrm{RN}$ : "Em quais momentos na sua atuação na unidade neonatal você percebe a presença de dor no RN prematuro?"; "Quais sinais você usa para identificar que um $\mathrm{RN}$ prematuro está sentindo dor?"; "O que você realiza quando percebe que um RN prematuro está sentindo dor?" e "O que a sua UTIN instituiu para avaliar a dor no $\mathrm{RN}$ prematuro?"

Os participantes lerem o Termo de Consentimento Livre e Esclarecido (TCLE) e o assinaram. A entrevista foi iniciada com auxílio de um dispositivo de áudio para gravação em espaço privativo e os dados foram transferidos para um microcomputador, onde foram transcritos e analisados. Cada entrevista durou em média 30 minutos, dentro da instituição hospitalar.

Para a análise das informações coletadas utilizou-se o método de Análise de Conteúdo, ${ }^{7}$ constituído por três etapas: a pré-análise (leitura profunda para atingir maior intimidade com o material selecionado); a exploração do material (identificação e seleção das principais falas e palavras-chave); e a interpretação (relação do material analisado de forma sintetizada com os objetivos e o problema levantado). ${ }^{7}$

O estudo foi aprovado pelo Comitê de Ética e Pesquisa com Seres Humanos sob o parecer $\mathrm{n}^{\circ} 2.060 .580$ e atendeu à Resolução 466/2012 do Conselho Nacional de Pesquisa em Saúde que trata de pesquisas envolvendo seres humanos.

Foram respeitados os princípios fundamentais da ética em pesquisa e o sigilo em relação à identidade e às informações fornecidas durante a entrevista. Pseudônimos foram utilizados com código "E" ("entrevistado") seguido de um número sequencial (por exemplo: E1, E2, E3) durante e ao final da entrevista, que foi realizada com presença de um entrevistador e do participante em um local reservado. 


\section{RESULTADOS E DISCUSSÃO}

Perfil sociocultural dos enfermeiros da UTIN

Todas as pessoas entrevistadas eram do sexo feminino (100\%). Entre elas, $71,4 \%$ eram especialistas em Neonatologia e $28,6 \%$ não possuíam nenhuma especialização. Quanto ao tempo de formação, 28,6\% obtiveram a graduação num período recente, de 0 a 5 anos, e $71,4 \%$ concluíram entre 5 e 10 anos. Sobre o tempo de atuação no setor, os dados obtidos mostraram que $71,4 \%$ das enfermeiras atuavam entre 0 a 5 anos, e que $28,6 \%$ apresentaram uma variação no tempo de atuação de 5 até 10 anos. Dentre as entrevistadas, $42,8 \%$ possuíam mais de uma especialização, sendo uma em Saúde Pública e duas em Urgência e Emergência.

Características como formação acadêmica suplementar e maior tempo de atuação no campo da neonatologia tem sido ligadas diretamente ao grau de conhecimento do profissional de saúde. ${ }^{8}$ Porém, mesmo possuindo a especialização na área, a ação do cuidado efetivo no alívio da dor pode ser reduzida frente à falta de prática e qualificação voltadas a esse aspecto. $^{8,9}$

A partir da análise emergiram-se duas categorias (C), a saber: 1 - Como o enfermeiro avalia a dor no RN prematuro; 2 - Ações do enfermeiro diante da dor no $\mathrm{RN}$ prematuro.
C1: Como o enfermeiro avalia a dor no recém-nascido prematuro

Quanto ao processo de avaliação da dor, o choro e a expressão facial foram os sinais mais presentes nas falas das enfermeiras da UTIN, associados a outros parâmetros como os fisiológicos. A avaliação desses últimos foi pouco abordada durante a pesquisa, pois as entrevistadas utilizaram a avaliação comportamental como a mais priorizada para a identificação da dor.

Os depoimentos ilustram a capacidade dos profissionais de enfermagem de identificar a dor, todavia, essa avaliação pode ser realizada tardiamente, quando os sinais presuntivos de dor já estão bastante avançados e o RN prematuro apresenta-se de maneira extremamente estressada:

Por expressão facial, choro, alteração nos sinais vitais, como frequência cardíaca, frequência respiratória, saturação (E1).

A gente percebe que ele está sentindo dor realmente, tá bem irritado (E2).

$\mathrm{O}$ RN geralmente demonstra um choro específico quando se provoca algum estimulo que cause dor. Embora não se tenham garantias de que o som emitido pelo bebê seja sempre de uma sensação dolorosa, pois há vários fatores que podem estar provocando o incômodo, tornando-se difícil na maioria das vezes a equipe 
identificar o motivo do choro somente através do som. ${ }^{4,10}$

Em relação à questão comportamental, as enfermeiras apresentaram uma associação com a intubação, onde os $\mathrm{RN}$ prematuros não conseguem se expressar através de sons e são os movimentos corporais que indicam o processo doloroso instalado.

Como a maioria dos pacientes de terapia intensiva são intubados, então a gente percebe questão comportamental dele, se tá se encolhendo (E4).

Associado a essa prática sonora, devem ser observados sinais na linguagem corporal da criança a fim de ter uma leitura mais precisa do que está motivando o desconforto. ${ }^{4}$

Quando eles sentem dor, eles normalmente fecham bastante as mãos como se estivessem apertando mesmo a mão pra demonstrar que estão sentindo dor (E7).

Um dos meios mais frequentes para avaliar o que provoca alteração no $\mathrm{RN}$ prematuro é a observação das expressões faciais, seguida dos movimentos corporais, sendo o choro, o alerta principal para que haja uma abordagem profissional do enfermeiro que deverá estar atento a todos os sinais emitidos pela criança sob os seus cuidados. ${ }^{10,11}$

Para a avaliação da dor no período neonatal pode-se utilizar parâmetros comportamentais e fisiológicos onde alguns sinais como frequência cardíaca e respiratória e saturação de oxigênio sofrem alterações no momento quando a dor ocorre. $^{4}$

Ficou perceptível na entrevista, o cuidado ao RN prematuro em relação aos estímulos externos e a consciência da maioria das entrevistadas em relação à sua fragilidade. Enquanto isso, outros profissionais mantêm o olhar voltado apenas para o desconforto gerado pelos dispositivos utilizados no RN prematuro:

"Muitas vezes a gente percebe lesões de pele, uma febre, choro irritante, luminosidade, na luz, quando irrita o olho do bebê, na verdade, o RN prematuro ele é muito sensível a qualquer estimulo (E6).

Quando a gente ta manipulando o paciente que tá usando algum determinado tipo de sonda, equipamento (E3).

$\mathrm{O}$ ato de manipular é classificado como cuidados realizados no $\mathrm{RN}$ que podem levar a um estresse e pode ser executado em qualquer horário. $\mathrm{O}$ excesso de movimentação no $\mathrm{RN}$ afeta o seu relaxamento, acarretando inúmeras alterações e estresse prematuro na qualidade de seu desenvolvimento. ${ }^{12}$

Vários fatores podem prejudicar a saúde do RN prematuro, portanto os cuidados e as técnicas de enfermagem devem garantir uma maneira de minimizar o risco e o desconforto do bebê enquanto ele permanecer na UTIN, a exemplo da incidência de luz excessiva que pode levar 
ao estresse, interferindo no seu desenvolvimento. ${ }^{10,13}$

Sabe-se que nas UTI os neonatos estão expostos a diversos procedimentos, dentre eles, alguns invasivos que causam dor e desestabilizam sua hemodinâmica, com destaque para a necessidade de punções, passagens de sondas orogástrica e vesical, realização de coleta de exames e intubação. Muitas vezes tais procedimentos ocorrem de forma traumática, apesar de todo cuidado para sua realização, trazendo tensão, agitação e incômodo. ${ }^{14}$

Diante de todas as ocasiões em que é possível reconhecer o processo doloroso, uma enfermeira citou que apenas durante a realização de procedimentos é provável que ela perceba essa dor, o que vai gerar uma avaliação e conduta precária no cuidado a esse RN prematuro.

\section{Durante procedimentos só (E5).}

Nota-se que existe uma preocupação quanto às necessidades de avaliação contínua no período de atuação na UTIN, quando é mencionada que não há um momento isolado para a percepção da dor, pois realizando uma observação constante, não existirão momentos em que $\mathrm{o} R \mathrm{RN}$ prematuro esteja sentindo dor e a equipe não perceba para intervir de forma adequada ou realize a prevenção necessária. ${ }^{8,9}$

"Quem trabalha na unidade de terapia intensiva na verdade tá em vigilância constante, então não tem um momento isolado pra perceber a dor do paciente (E4).

O cuidado de enfermagem torna-se prioritário na atenção ao neonato, buscando uma assistência voltada à humanização por meio de um olhar minucioso, de forma a estar sempre alerta às necessidades do RN prematuro. Faz-se necessário contribuir no seu desenvolvimento adequado e na implementação de ações voltadas à assistência. $^{8}$

As enfermeiras com mais experiência são capazes de identificar se o choro ocorre em razão da fome, da dor ou de eliminações. Sabe-se que o choro de dor tem uma forma peculiar e característica, com isso, profissionais que não possuem tanto tempo na assistência podem apresentar dúvidas acerca das particularidades que esses sinais podem apresentar e não saber a quê ele se destina. ${ }^{9,10}$

Então, a gente como profissional tem que saber o momento certo, ter uma visão ampla de quando o bebê ele tá bem, tá calmo, confortável e quando o bebê ele tá sentindo dor. E é muito fácil, pra quem tem experiência, perceber, o choro é diferente de um choro de sono, em choro de fome, de um choro que tá de xixi, o choro é diferente (E6).

A gente percebe a presença mais em relação à irritabilidade, choro, a gente assim cem por cento não tem, certo? (E2).

A habilidade demonstrada no momento de realizar a identificação da dor 
é influenciada pela competência técnica e o tempo de experiência na área, pois o fato do RN não poder se expressar verbalmente, faz com que suas queixas sejam expressas através de sinais emitidos pela criança. ${ }^{11,13}$

A percepção da dor é feita através da mudança do perfil fisiológico e comportamental do $\mathrm{RN}$ por meio de estímulos realizados. A identificação das situações que causam dor fará com que o profissional busque estratégias para o alívio dessa dor. ${ }^{15}$

Devido à ausência de protocolo na unidade onde foi realizada a pesquisa, a mensuração da dor torna-se superficial e são utilizados parâmetros para analisar a dor, sinais que, quando observadas de forma fragmentada e aleatória, não trazem tanta confiabilidade.

Alguns profissionais destacaram que, mesmo com existência de alguns métodos de avaliação da dor, essa conduta ainda é pouco utilizada e que se aguarda a implantação de uma escala própria para que a assistência seja sistematizada.

A gente utiliza muito pouco os métodos pra avaliar a dor, que a gente tem da escala, tem da face, entre outros. A gente tá usando muito pouco (E3).

A gente tá com projeto de escala da dor, tá em curso já (E5).

Para identificar as várias formas de avaliar a dor no neonato, foram criadas escalas de avaliação para que se pudesse de forma mais objetiva, com parâmetros específicos, perceber os momentos que causam dor no RN e, através disso, intervir de forma eficaz. A aplicação dessas escalas minimiza os erros perante situações que causam dor e que muitas vezes são imperceptíveis. A associação de fatores fisiológicos e comportamentais representa uma análise fidedigna dessa avaliação. ${ }^{15,16}$

Na verdade, a gente da UTI, a gente já tem essa escala, que é uma escala pra avaliar o grau de dor do prematuro (E6).

A gente tem a escala de NIPS né, que aí é feita a avaliação diária (E7).

Nós utilizamos da escala que é a NIPS, que geralmente é utilizada pela maioria dos profissionais (E1).

As escalas multidimensionais, como a Neonatal Infant Pain Scale (NIPS), foram criadas com o principal objetivo de interpretar os sinais comportamentais e fisiológicos apresentados pelo RN, facilitar e orientar a equipe em ações de prevenção e manejo da dor. ${ }^{6}$

É perceptível o desconhecimento de alguns enfermeiros sobre os parâmetros utilizados na escala, ao defini-la apenas como um instrumento para avaliar o grau de dor.

C2: Ações do enfermeiro diante da dor no $\mathrm{RN}$ prematuro

Houve destaque para diversas técnicas de alívio da dor, englobando tanto as medidas farmacológicas como as não 
farmacológicas, e a preferência por um determinado método.

Durante a realização de procedimentos menos invasivos como as punções venosas, coletas de material sanguíneo e aspiração de vias aéreas, os manejos não farmacológicos de amenizar a dor podem ser utilizados, tais como: sucção nutritiva, utilização de glicose, promoção de ambiente confortável e método Canguru. Esses artifícios favorecem a redução do tempo de choro, melhora da oxigenação e do gasto energético, promovendo descanso e analgesia. ${ }^{17,18}$

Em casos de procedimentos mais complexos ou que possam gerar um estímulo duradouro e intenso, é recomendada uma intervenção farmacológica, que acontece através do uso de anti-inflamatórios, opioides e anestésicos de efeito local. Além disso, esses métodos também podem ser utilizados de forma associada para potencializar o alívio. ${ }^{17,18}$

Foi evidenciada a necessidade do uso de abordagem preventiva sempre que a dor possa desencadear-se. No caso de procedimentos como punções venosas, entre outros, é melhor fazer a prevenção do que esperar o estímulo doloroso ocorrer para poder intervir ou aliviar.
A gente percebe essa percepção de dor no prematuro no momento de uma punção venosa (E6).

Os métodos não farmacológicos foram os mais citados pelas entrevistadas e mostraram ser empregados durante a realização da rotina da unidade neonatal, tais como os observados no relato a seguir:

A gente tem outras medidas também... não farmacológicas que são mudança de decúbito, toque terapêtico, pra incentivar a melhora desse bebê (E1).

Houve preocupação quanto ao conforto proporcionado ao RN prematuro, destacando o entendimento da equipe frente à necessidade de um cuidado diferenciado para esse paciente.

Diversos profissionais citaram a glicose como forma de redução do estímulo doloroso, além de defender que essa seja a forma mais eficaz de obter tal resultado. Isso demonstra conhecimento sobre os efeitos da utilização dessa substância para diminuição e/ou impedimento do efeito doloroso.

Primeiro a gente oferece a glicose que ajuda a diminuir a dor (E5).

A gente usa basicamente glicose a vinte $e$ cinco por cento pra evitar que ele sinta dor (E2).

A utilização de glicose é baseada na estimulação de opioides endógenos, neurotransmissores fisiológicos que regulam a sensação de dor, fazendo com que o RN se acalme antes da realização de um procedimento. ${ }^{9}$ 
Coloca ele como se fosse dentro de um charutinho, com o próprio lençol a gente enrola ele, pra ele ficar calmo, aconchegado (E7).

$\mathrm{O}$ recurso de envolver o recémnascido em um ambiente mais aconchegante tem a finalidade de transmitir ao mesmo o sentimento de maior segurança durante a realização dos procedimentos. $^{8}$

Apesar de não ser o método de escolha mais citado, os recursos farmacológicos mostraram-se presentes no cotidiano da unidade, sendo em alguns momentos os principais para redução da dor.

A gente comunica ao médico e vê se faz uma dipirona endovenosa se ele tiver com algum acesso (E2).

A gente avalia todo um aspecto, porque se é uma dor, uma lesão, a gente comunica ao médico, ele passa um analgésico (E6).

A variação na utilização de recursos farmacológicos e não farmacológicos demonstra que as enfermeiras têm ciência das diversas intervenções que podem ser colocadas em prática para proporcionar ao RN uma melhor qualidade de vida durante a internação. Em contrapartida, não existe padronização das atividades exercidas para o controle da dor.

As falas das entrevistadas revelam que algumas profissionais ainda sentem dificuldades em tratar a dor:

É baseado unicamente prescrição médica, se não tiver nada prescrito, informar ao médico, ele vem avaliar e prescreve medicação (E4).
Nós estamos implantando aqui na UTI um grupo de dor já específico pra que enfermeiros foquem justamente nisso, a gente crie escalas, crie estratégias pra melhorar isso no recém-nascido, mas não implantamos ainda (E1).

Fica evidente que a falta de um protocolo faz com que cada profissional desenvolva sua própria rotina, escolhendo o método que lhe pareça mais eficaz. Assim, muitas técnicas importantes que deveriam ser implementadas na prática do cuidado diário, podem deixar de ser utilizadas devido à supervalorização da prescrição ou conduta médica. Além disso, parece existir uma imprecisão no papel de cada especialidade profissional nesse âmbito do cuidado:

A nossa equipe tá restrita realmente à prescrição médica, essa comunicação com o médico de perceber a dor do paciente, sinalizar e aí a gente vai cuidar. Mas quando o protocolo tiver pronto, então a equipe de enfermagem vai ter uma certa autonomia de ter um comportamento um pouco diferente até o médico chegar. Vou ter um norte ali do que fazer, mas no momento é só a prescrição médica mesmo (E4).

Sabe-se que o tratamento adequado da dor neonatal está associado a menores efeitos e diminuição da mortalidade. Diante disso, é importante a utilização de técnicas de prevenção e controle da dor na UTIN. ${ }^{12,15,16,18}$

As enfermeiras identificam os procedimentos que causam dor no RN prematuro e algumas se preocupam em utilizar estratégias para minimizar os 
efeitos nocivos da mesma. Como outra forma de estar minimizando e tratando esse processo doloroso, foi citada a importância do contato familiar e da equipe multidisciplinar para que esse cuidado se torne eficaz, o que acentua a sensibilização dos profissionais para a expressão do $\mathrm{RN}$ prematuro, com a finalidade de melhorar a assistência desses pacientes, que são submetidos a inúmeros procedimentos dolorosos ao longo das internações.

A gente já tem essa maneira de acalentar, de dá um carinho que ele precisa. A gente sabe quando o bebê, só um carinhozinho que é de pai, que é de mãe, que é da equipe toda né [...] a criança na verdade que tá na UTI ela é carente de família, ela é carente de carinho, carente de tudo, de todos os aspectos. Então a gente tem que se preocupar com tudo na verdade (E6).

O controle eficaz da dor pede a atuação de uma equipe multidisciplinar, pois cada indivíduo oferece os recursos que possui na resolução dos problemas. É imprescindível distinguir o que cada especialidade profissional precisa saber nesse tipo de atuação e melhorar a qualidade do cuidado prestado., ${ }^{9,18}$

\section{CONCLUSÕES}

Este estudo se mostrou importante a fim de valorizar os discursos das pessoas que estão diretamente envolvidas nesse processo do cuidado ao RN prematuro. Conclui-se que, na equipe de enfermagem, ainda existe uma grande distância entre o conhecimento teórico, o uso de escalas e a conduta prática quanto à avaliação e manejo da dor de recém-nascidos prematuros. Percebeu-se que as enfermeiras com especialização na área e maior tempo de formação acadêmica demonstraram nível de conhecimento mais elevado e segurança acerca do tema. Todavia, não há padronização na avaliação e no manejo da dor dos neonatos no local estudado.

O estudo apresenta como limitação ser uma pesquisa locorregional desenvolvida em uma maternidade de referência que não havia recursos e serviços essenciais para avaliar a dor no RN prematuro, algo que talvez não possa ser generalizado às demais realidades. Por isso é oportuno que outros estudos sejam desenvolvidos nas diversas regiões do país.

Porém, essa pesquisa aponta a potencialidade de ser um estudo inédito na área da saúde neonatal na região sobre a temática, fornecendo informações das percepções das situações vivenciadas pelos enfermeiros no cuidado ao RN prematuro.

Como implicações para a prática, destacam-se o estabelecimento de protocolos de dor, como o uso das escalas para avaliação e manejo adequado da dor do prematuro, assim como estratégias educativas com a equipe de enfermagem para sensibilização da dor. Isso traria benefícios para mensuração, mais 
especificidade nas funções de cada profissional, autonomia e independência nas práticas desempenhadas pelo enfermeiro.

Fonte de financiamento: nenhuma.

\section{REFERÊNCIAS}

1. Chaves ACF, Santos AP, Ataíde KMN, Cunha KJB. Cuidado e manutenção da integridade da pele do neonato prematuro. Rev Enferm UFPE on line. [Internet]. 2019 [citado em 29 jul 2021]; 13(2):378-84. Disponível em: https://periodicos.ufpe.br/revistas/revistaen fermagem/article/view/237974. doi: https://doi.org/10.5205/1981-8963v13i2a237974p378-384-2019

2. Chuang L, Wang S, Ma M, Lin C, Chen C, Huang M. A modified developmental care bundle reduces pain and stress in preterm infants undergoing examinations for retinopathy of prematurity: a randomised controlled trial. J Clin Nurs. [Internet]. 2019 [citado em 29 jul 2021]; 28(3-4):545-59. Disponível em: https://pubmed.ncbi.nlm.nih.gov/30091495 /. doi: 10.1111/jocn.14645.

3. Bonutti DP, Daré MF, Castral TC, Leite AM, Vici-Maia JA, Scochi CGS.

Dimensioning of painful procedures and interventions for acute pain relief in premature infants. Rev Latinoam Enferm. [Internet]. 2017 [citado em 29 jul 2021]; 25:e2917. Disponível em: https://www.scielo.br/j/rlae/a/SZDJpChvvx ZvNtqD6H3vgvD/?lang=em. doi: https://doi.org/10.1590/15188345.1387.2917

4. Maciel HIA, Costa MF, Costa ACL, Marcatto JO, Manzo BF, Bueno M. Medidas farmacológicas e não farmacológicas de controle e tratamento da dor em recém-nascidos. Rev Bras Ter Intensiva. [Internet]. 2019 [citado em 29 jul 2021]; 31(1):21-6. Disponível em:
https://www.scielo.br/j/rbti/a/WDnJF38dg pWWwwmwrDFStdP/?format=pdf\&lang= pt. doi: https://doi.org/10.5935/0103507X.20190007

5. Marques ACG, Lamy ZC, Garcia JBS, Gonçalves LLM, Bosaipo DS, Silva HDC, et al. Avaliação da percepção de dor em recém-nascidos por profissionais de saúde de unidade neonatal. Cad Saúde Colet. [Internet]. 2019 [citado em 29 jul 2021]; 27(4):432-36. Disponível em: https://www.scielo.br/j/cadsc/a/yTSPw96z S4MWhkB5HqFqMCx/?lang=pt. doi: https://doi.org/10.1590/1414462X201900040156

6., Silva JSLG, Magalhães SGS. O cuidado humanizado na Unidade de Terapia Intensiva Neonatal. Rev Pró-UniverSUS. [Internet]. 2019 [citado em 29 jul 2021]; 10(1):129-32. Disponível em: http://editora.universidadedevassouras.edu. br/index.php/RPU/article/view/1640. doi: https://doi.org/10.21727/rpu.v10i1.1640 7. Minayo MCS. Pesquisa social: teoria, método e criatividade. 31. ed. Petrópolis: Vozes; 2012.

8. Câmara TL, Pereira NCST, Silva RM da. Enfermagem e o manuseio do recém nascido na Unidade de Terapia Intensiva Neonatal. Rev Uningá. [Internet]. 2019 [citado em 29 jul 2021]; 56(S2):222-33. Disponível em:

http://revista.uninga.br/index.php/uninga/ar ticle/view/2156.

9. Christoffeli MM, Castral TC, Daré MF, Montanholi LL, Gomes ALM, Scoch CGS. Atitudes dos profissionais de saúde na avaliação e tratamento da dor neonatal. Esc Anna Nery Rev Enferm. [Internet]. 2017 [citado em 29 jul 2021]; 21(1):1-8.

Disponível em: https://www.scielo.br/j/ean/a/JFQ4N4gDZ NN44q3kFD8dfjv/?lang=pt doi: https://doi.org/10.5935/14148145.20170018 10. Hall RW, Anand KJS. Pain management in newborns. Clin Perinatol. [Internet]. 2014 [citado em 29 jul 2021]; 41(4):895-924. Disponível em: https://www.ncbi.nlm.nih.gov/pmc/article 
s/PMC4254489/. doi:

10.1016/j.clp.2014.08.010

11. Moretto LCA, Perondi ER, Trevisan

MG, Teixeira GT, Hoesel TC, Costa LD.

Dor no recém-nascido: perspectivas da

equipe multiprofissional na unidade de

terapia intensiva neonatal. Arq Ciências

Saúde UNIPAR. [Internet]. 2019 [citado em 29 jul 2021]; 23(1):29-34. Disponível em:

https://www.revistas.unipar.br/index.php/s aude/article/view/6580. doi:

https://doi.org/10.25110/arqsaude.v23i1.20 19.6580

12. Levy J, Hassan F, Plegue MA,

Sokoloff MD, Kushwaha JS, Chervin RD, et al. Impact of hands-on care on infant sleep in the neonatal intensive care unit. Pediatr Pulmonol. [Internet]. 2017 [citado em 29 jul 2021]; 52(1):84-90. Disponível em:

https://pubmed.ncbi.nlm.nih.gov/27362468

/. doi: https://doi.org/10.1002/ppul.23513. 13. Silva AR, Hofmann E, Zancaron SS. Acolhimento na Unidade de Terapia Intensiva Neonatal: percepções das profissionais e mães. Argum. [Internet]. 2018 [citado em 29 jul 2021]; 10(1):198212. Disponível em:

https://dialnet.unirioja.es/servlet/articulo?c odigo $=6545997$.

14. Suleiman ZA, Kolawole IK,

Adegboye KA, Adeboye MA,

Onuchukwuet CE, Bello S, et al.

Analgesia for procedural pain: Current

practice among Nigerian paediatricians.

Port Harcourt Med J. [Internet]. 2019

[citado em 29 jul 2021]; 13(1):13-8.

Disponível em:

https://www.phmj.org/article.asp?issn=07

95-

3038; year $=2019 ;$ volume $=13$; issue $=1$; spag

$\mathrm{e}=13$; epage $=18$; aulast $=$ Suleiman. doi:

https://doi.org/10.4103/phmj.phmj_3_19

15. Oliveira CR de, Santos JMJ, Guarda

LEA, Barbieratto BJ, Dare MF, Leonello

DCB, et al. Manejo da dor neonatal em

uma maternidade de risco habitual:

perspectivas de profissionais líderes da

equipe de saúde. REME Rev Min Enferm.
[Internet]. 2020 [citado em 29 jul 2021];

24:e-1289. Disponível em:

http://reme.org.br/artigo/detalhes/1435.

doi: http://www.dx.doi.org/10.5935/1415-

2762.20200018

16. Almeida CR, Morais AC, Lima KDF, Silva ACOC. Cotidiano de mães acompanhantes na unidade de terapia intensiva neonatal. Rev Enferm UFPE on

line. [Internet]. 2018 [citado em 29 jul 2021]; 12(7):1949-56. Disponível em: https://pesquisa.bvsalud.org/portal/resourc e/pt/biblio-986691

17. Simons SHP, Dijk M, Anand KS, Roofthooft D, Lingen RA, Tibboel D. Do we still hurt newborn babies? A prospective study of procedural pain and analgesia in neonates. Arch Pediatr Adolesc Med. [Internet]. 2003 [citado em 29 jul 2021]; 157(11):1058-64. Disponível em:

https://pubmed.ncbi.nlm.nih.gov/1460989 3/ doi:

https://doi.org/10.1001/archpedi.157.11.1 058

18. Damaceno AN, Assumpção PK, Belmonte GPS. Avaliação da dor do recém-nascido pela equipe de enfermagem: scoping review. Rev Enferm Atenção Saúde [Internet]. 2019 [citado em 29 jul 2021]; 8(2):135-48. Disponível em: http://seer.uftm.edu.br/revistaeletronica/ind ex.php/enfer/article/view/2405. doi: https://doi.org/10.18554/reas.v7i2.2405

RECEBIDO: $14 / 02 / 2020$

APROVADO: $10 / 02 / 2021$

PUBLICADO: 09/2021 